\title{
Numerical solution and stability analysis of the Sine-Gordon system in two dimensions
}

\author{
Saad A. Manna \\ College of Science \\ Zakho University
}

\section{Received on:27/01/2009}

Haneen T. Jassim

College of Computer Science and

Mathematics

University of Mosul

Accepted on:17/05/2009

\section{ABSTRACT}

This paper deals with the numerical solution for Sine-Gordon system in two dimensions using two finite difference methods the (ADE) and (ADI) methods .A comparison between the two methods has been done and we have obtained that the (ADE) method is the easer while the (ADI) method is more accurate than the (ADE) method. We also studied the stability analysis for each method by using Fourier (Von Neumann) method and we have obtained that the (ADI) method is unconditionally stable while the (ADE) method is stable under the condition $r^{2} \leq \frac{1}{2 c^{2}}$ and $r^{2} \leq \frac{1}{2}$ where $c^{2}$ is the ratio of the waves speed $\mathrm{u}, \mathrm{w}$ and $r^{2}=(\Delta t)^{2} /(\Delta x)^{2}$.

Keywords: finite difference methods, Sine-Gordon System.

$$
\begin{aligned}
& \text { الحل العددي وتحليل الاستقرارية لنظام Sine-Gordon في بعدين } \\
& \text { حنين طلال جاسم } \\
& \text { كلية علوم الحاسوب والرياضيات } \\
& \text { جامعة الموصل }
\end{aligned}
$$

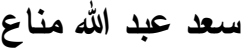

$$
\begin{aligned}
& \text { كلية العلوم }
\end{aligned}
$$

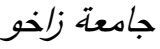

$$
\begin{aligned}
& \text { تاريخ قبول البحث: 2009/05/17 } \\
& \text { تاريخ استلام البحث: 2009/01/27 }
\end{aligned}
$$

الكلمات المفتاحية: طرائق الفروقات المنتهية , نظام Sine-Gordon . 


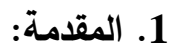

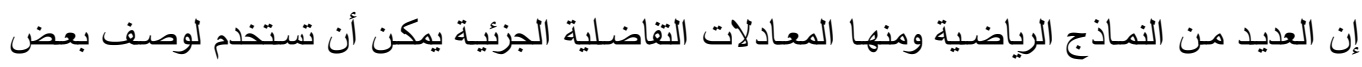

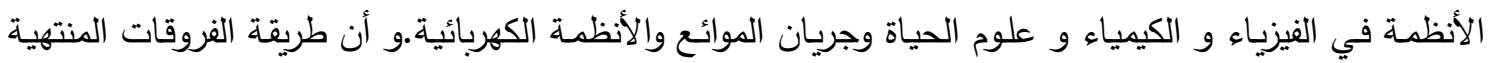
هي الأكثر استخداما وشيوعا وبشكل تفصيلي لحل صيخ مختلفة لهذه ونهان (Finite Differences Method)

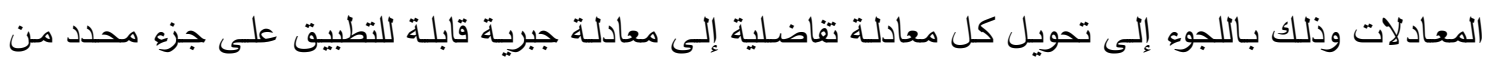
المجال المتضمن حل المعادلة التفاضلية. [1] الحل العددي للمعادلة التفاضلية الجزئية هو إيجاد حل المعادلات التفاضلية الجزئية بالتعويض عن المعادلة التفاضلية بمعادلة تقريبية ثم حلها، إذ أن طريقة تقريب الفروقات المنتهية تعوض عن المشتقات الجزئية بالفروقات التهنية

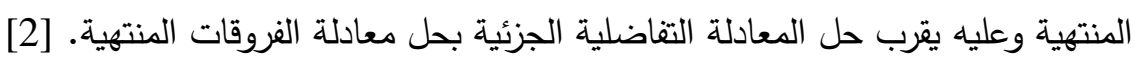
وضعت المعادلة Klein-Gordon من قبل العالمين Klein and Gordon في العشرينيات بوصفها يحله نموذجا لمعادلة الموجة اللاخطية، وأول من أطلق اسم Sine-Gordon عليها العالم Kruskal، إذ أن (x,t

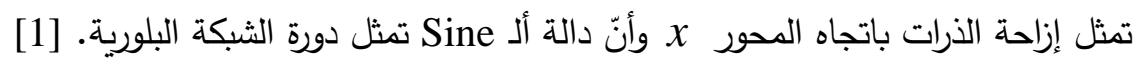
ودرس كل من Ablowitz, Herbst and Schober عام (1996) السلوك العددي للتقطيع القابل للتكامل التام و المضاعف لمعادلة Sine-Gordon ، إذ بينوا انه يسمح لقيم ابتدائية معينة بعدم الاستقرار على شكل تذبذبات في

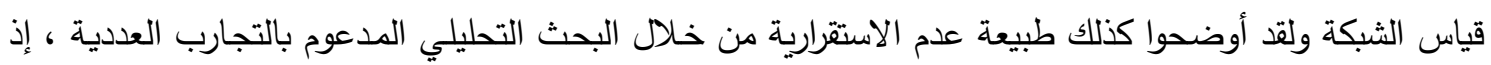

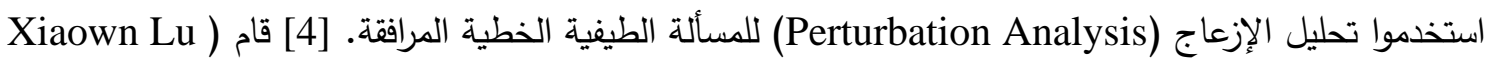
Symplectic عام (1998) بدراسة نظام معادلات Sine-Gordon (and Rudolf Schmid

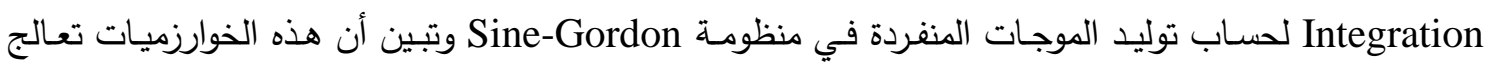
البناء العام والاستقرار التبولوجي للموجات المنفردة بشكل جيد. [9] كما درس Jesus Adrian and Espinola Rocha عام (2000) تحويل منظومة Klein-Gordon من منظومة معادلات تفاضلية جزئية إلى نظام ديناميكي

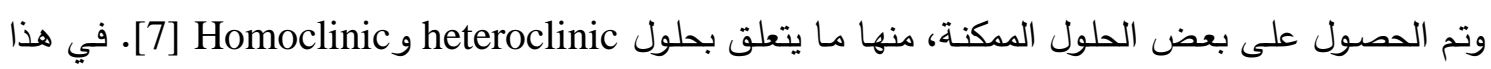
البحث سوف نقوم بتطبيق طريقة تقريبات الفروقات المنتهية لنظام Sine-Gordon في بعدين باستخدام طريقتين

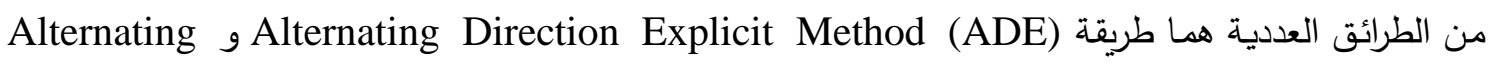
كما سنقوم بدراسة استقرارية الحل العددي لكل من الطريقتين والمقارنة

إن نظام Sine-Gordon في بعدين يكون بالثكل الآتي [8] :

$$
\left.\begin{array}{l}
\frac{\partial^{2} u}{\partial t^{2}}-\frac{\partial^{2} u}{\partial x^{2}}-\frac{\partial^{2} u}{\partial y^{2}}=-\delta^{2} \sin (u-w) \\
\frac{\partial^{2} w}{\partial t^{2}}-c^{2} \frac{\partial^{2} w}{\partial x^{2}}-c^{2} \frac{\partial^{2} w}{\partial y^{2}}=\sin (u-w)
\end{array}\right\}
$$

على المنطقة المستطيلة 


$$
\begin{aligned}
& u(x, y, 0)=f(x, y), \frac{\partial u(x, y, 0)}{\partial t}=0,0 \leq x, y \leq 2 \pi \\
& w(x, y, 0)=g(x, y), \frac{\partial w(x, y, o)}{\partial t}=0,0 \leq x, y \leq 2 \pi
\end{aligned}
$$

والثرط الحدودي:

3. اشتقاق صيغة طريقة الـ Alternating Direction Explicit (ADE) لنظام Sine-Gordon: تعرف الإحداثيات (x,y,t) على نقاط الشبكة بالثكل الآتي: $x=n h, \quad y=m k, t=p z, n, m=1,2, \ldots . ., M, \quad p=1,2, \ldots . ., N$

[5] إذ أن $h=\Delta x, k=\Delta y, z=\Delta t$ [ هي أعداد صحيحة وعليه فإنّ نقاط w,u على الشبكة تكون بالشكل الآتي:

$u(n h, m k, p z)=u_{n, m, p}$

$w(n h, m k, p z)=w_{n, m, p}$

إن التقريب العددي للمشتقات الجزئيـة الأولى والثانيـة للدالـة (u) بالنسبة إلى (x) و و (t) والتي حصلنا عليها

$$
\begin{aligned}
& \left(\frac{\partial u}{\partial x}\right)_{n, m}=\frac{u_{n+1, m}-u_{n, m}}{h} \\
& \left(\frac{\partial u}{\partial t}\right)_{n, m}=\frac{u_{n, m+1}-u_{n, m}}{k} \\
& \left(\frac{\partial^{2} u}{\partial x^{2}}\right)_{n, m}=\frac{u_{n+1, m}-2 u_{n, m}+u_{n-1, m}}{h^{2}} \\
& \left(\frac{\partial^{2} u}{\partial t^{2}}\right)_{n, m}=\frac{u_{n, m+1}-2 u_{n, m}+u_{n, m-1}}{k^{2}}
\end{aligned}
$$

باستخدام مفكوك تايلر هي كما يلي [3]:

وباستخدام المعادلتين (4) و(5) بالنسبة للمتغيرين w و ف فإن النظام (1) يصبح:

$$
\begin{aligned}
& \frac{u_{n, m, p+1}-2 u_{n, m, p}+u_{n, m, p-1}}{z^{2}}=-\delta^{2} \sin \left(u_{n, m, p}-w_{n, m, p}\right)+\frac{u_{n+1, m, p}-2 u_{n, m, p}+u_{n-1, m, p}}{h^{2}} \\
& +\frac{u_{n, m+1, p}-2 u_{n, m, p}+u_{n, m-1, p}}{k^{2}} \\
& \frac{w_{n, m, p+1}-2 w_{n, m, p}+w_{n, m, p-1}}{z^{2}}=\sin \left(u_{n, m, p}-w_{n, m, p}\right)+c^{2} \frac{w_{n+1, m, p}-2 w_{n, m, p}+w_{n-1, m, p}}{h^{2}} \\
& +c^{2} \frac{w_{n, m+1, p}-2 w_{n, m, p}+w_{n, m-1, p}}{k^{2}} \\
& u_{n, m, p+1}=-z^{2} \delta^{2} \sin \left(u_{n, m, p}-w_{n, m, p}\right)-u_{n, m, p-1}+\left(2-4 r^{2}\right) u_{n, m, p} \\
& \text { • } k=h, r=z / h \text { لتكن }
\end{aligned}
$$




$$
\begin{array}{r}
+r^{2}\left(u_{n+1, m, p}+u_{n-1, m, p}+u_{n, m+1, p}+u_{n, m-1, p}\right) \\
w_{n, m, p+1}=z^{2} \sin \left(u_{n, m, p}-w_{n, m, p}\right)-w_{n, m, p-1}+\left(2-4 c^{2} r^{2}\right) w_{n, m, p} \\
+c^{2} r^{2}\left(w_{n+1, m, p}+w_{n-1, m, p}+w_{n, m+1, p}+w_{n, m-1, p}\right)
\end{array}
$$

إن المعادلتين (6) و(7) هما صيغة طريقة الـ Sine-Gordon نلاحظ أنهما تحتاجان إلى توفر

$$
\text { ست نقاط في المستوي الن المعادين }
$$

لأجل حساب القيم يجب أن نجد قيم النقاط في المستوي الثاني،نعوض عن p=1 p في المعادلتين (6) و(7)

$$
\begin{aligned}
u_{n, m, 2}=-z^{2} \delta^{2} \sin \left(u_{n, m, 1}-w_{n, m, 1}\right)-u_{n, m, 0} & +\left(2-4 r^{2}\right) u_{n, m, 1} \\
& +r^{2}\left(u_{n+1, m, 1}+u_{n-1, m, 1}+u_{n, m+1,1}+u_{n, m-1,1}\right) \\
w_{n, m, 2}=z^{2} \sin \left(u_{n, m, 1}-w_{n, m, 1}\right)-w_{n, m, 0}+ & \left(2-4 c^{2} r^{2}\right) w_{n, m, 1}
\end{aligned}
$$$$
\text { فنحصل على: }
$$

$$
+c^{2} r^{2}\left(w_{n+1, m, 1}+w_{n-1, m, 1}+w_{n, m+1,1}+w_{n, m-1,1}\right)
$$

وباستخدام الفروقات المركزية في تطبيق الثروط الابتدائية نحصل على:

$$
\frac{u_{n, m, 2}-u_{n, m, o}}{2 k}=0 \Rightarrow u_{n, m, 2}=u_{n, m, 0}
$$

$$
\frac{w_{n, m, 2}-w_{n, m, o}}{2 k}=0 \Rightarrow w_{n, m, 2}=w_{n, m, 0}
$$

وبتعويض المعادلتين (10) و(11) في المعادلتين (8) و(9) وبالترتيب ينتج:

$$
\begin{aligned}
u_{n, m, 2}=\frac{1}{2}\left[-z^{2} \delta^{2} \sin \left(u_{n, m, 1}-w_{n, m, 1}\right)+\right. & \left(2-4 r^{2}\right) u_{n, m, 1} \\
& \left.+r^{2}\left(u_{n+1, m, 1}+u_{n-1, m, 1}+u_{n, m+1,1}+u_{n, m-1,1}\right)\right]
\end{aligned}
$$

$$
\begin{aligned}
w_{n, m, 2}=\frac{1}{2}\left[z^{2} \sin \left(u_{n, m, 1}-w_{n, m, 1}\right)+\right. & \left(2-4 c^{2} r^{2}\right) w_{n, m, 1} \\
& \left.+c^{2} r^{2}\left(w_{n+1, m, 1}+w_{n-1, m, 1}+w_{n, m+1,1}+w_{n, m-1,1}\right)\right]
\end{aligned}
$$

4. اشتقاق صيغة طريقة Alternating Direction Implicit (ADI) لنظام Sine-Gordon: هذه الطريقـة تم تطويرهـا مـن قبل العـالمين (Peaceman) و (Rachford) عـام 1955 [10]، وهـي تتطلب خزنا قليلاً في الحاسبة كما أنها طريقة دقيقة. تشمل الطريقة تعاقب خطوتين مختلفتين من تقريبات الفروقات المنتهية في بعدين.

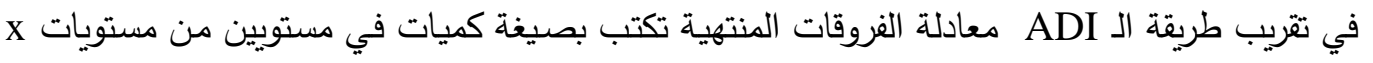
وبشكل عام فإنّ تقريبين من تقريبات الفروقات المنتهية استخدمت بالتعاقب، احدها لحساب التقدم من المستوي

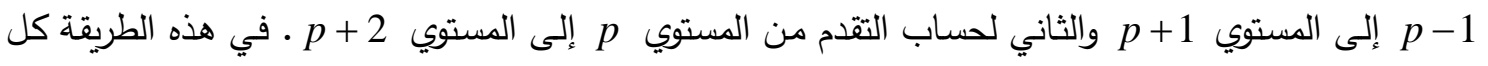
من الخطوتين تثمل الحركة في كل من الاتجاهين x و و ، ففي الخطوة الأولى الحركة في الاتجاه $x$ وتصاغ

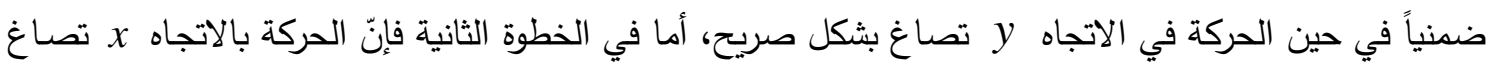

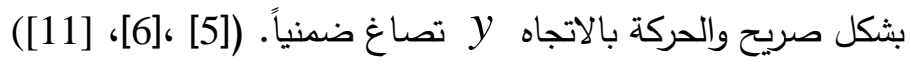


في الخطوة الأولى يمكن تمثيل الحركة من المستوي 1-p إلى المستوي 1 1 1 باستبدال الفروقات المنتهية الضمنية عند المستوي 1 المستوي 1- 1 وكما يأتي:-

$\frac{u_{n, m, p+1}-2 u_{n, m, p}+u_{n, m, p-1}}{z^{2}}=-\delta^{2} \sin \left(u_{n, m, p}-w_{n, m, p}\right)+\frac{u_{n+1, m, p+1}-2 u_{n, m, p+1}+u_{n-1, m, p+1}}{h^{2}}$

$$
+\frac{u_{n, m+1, p-1}-2 u_{n, m, p-1}+u_{n, m-1, p-1}}{k^{2}}
$$

$\frac{w_{n, m, p+1}-2 w_{n, m, p}+w_{n, m, p-1}}{z^{2}}=\sin \left(u_{n, m, p}-w_{n, m, p}\right)+c^{2}\left(\frac{w_{n+1, m, p+1}-2 w_{n, m, p+1}+w_{n-1, m, p+1}}{h^{2}}\right)$

$$
+c^{2}\left(\frac{w_{n, m+1, p-1}-2 w_{n, m, p-1}+w_{n, m-1, p-1}}{k^{2}}\right)
$$

$\left(1+2 r^{2}\right) u_{n, m, p+1}-r^{2}\left(u_{n+1, m, p+1}+u_{n-1, m, p+1}\right)=-z^{2} \delta^{2} \sin \left(u_{n, m, p}-w_{n, m, p}\right)$

$$
-\left(1+2 r^{2}\right) u_{n, m, p-1}+r^{2}\left(u_{n, m+1, p-1}+u_{n, m-1, p-1}\right)+2 u_{n, m, p}
$$

$\left(1+2 c^{2} r^{2}\right) w_{n, m, p+1}-c^{2} r^{2}\left(w_{n+1, m, p+1}+w_{n-1, m, p+1}\right)=z^{2} \sin \left(u_{n, m, p}-w_{n, m, p}\right)$

$$
-\left(1+2 c^{2} r^{2}\right) w_{n, m, p-1}+c^{2} r^{2}\left(w_{n, m+1, p-1}+w_{n, m-1, p-1}\right)+2 w_{n, m, p}
$$

المعادلات(14) و (15) تمثل صيغة ألـ (ADI) لنظام Sine-Gordon، أما بالنسبة للنقاط في المستوي 1+

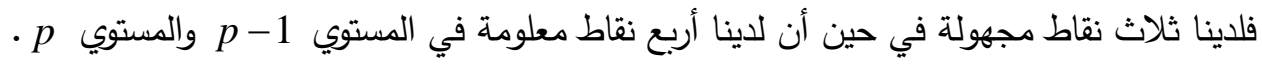
وللبدء في إيجاد الحسابات نحتاج إلى إيجاد الحسابات في المستوي الثاني وسوف نستخدم تقريب الفروقات الأمامية للشرط الابتدائي

$\frac{u_{n, m, 2}-u_{n, m, 1}}{z}=0 u_{n, m, 2}=u_{n, m, 1} \quad \Rightarrow$

$\frac{w_{n, m, 2}-w_{n, m, 1}}{z}=0 \quad \Rightarrow w_{n, m, 2}=w_{n, m, 1}$

$\mathrm{AX}=\mathrm{B}$

المعادلات (14) و(15) تكوّن نظام جبري ثلاثي الأقطار

$\mathrm{VX}=\mathrm{C}$

الثروط الحدودية في المعادلة الأولى والأخيرة وكما يأتي:-

$$
\begin{array}{ll}
u_{1, m, p+1}=0 & \\
w_{1, m, p+1}=0 & u_{M, m, p+1}=0 \\
w_{M, m, p+1}=0
\end{array}
$$

أمسا في الخطوة الثانيـة فتمثل الحركـة مـن المستوي p p إلى المستوي 2+ الفروقات المنتهية الصريحة عند المستوي p واستبدال $p \frac{\partial^{2} u}{\partial y^{2}}$ وبتقريب الفروقات المنتهية الضمنية عند المستوي 2+p وكما يأتي :- 


$$
\begin{gathered}
\begin{array}{c}
\frac{u_{n, m, p+2}-2 u_{n, m, p+1}+u_{n, m, p}}{z^{2}}= \\
+\frac{u_{n+1, m, p}-2 u_{n, m, p}+u_{n-1, m, p}}{h^{2}}+\frac{u_{n, m+1, p+2}-2 u_{n, m, p+2}+u_{n, m-1, p+2}}{k^{2}} \\
\left.\begin{array}{c}
\frac{w_{n, m, p+2}-2 w_{n, m, p+1}+w_{n, m, p}}{z^{2}}= \\
\sin \left(u_{n, m, p}-w_{n, m, p}\right) \\
+c^{2}\left(\frac{w_{n+1, m, p}-2 w_{n, m, p}+w_{n-1, m, p}}{h^{2}}\right)+c^{2}\left(\frac{w_{n, m+1, p+2}-2 w_{n, m, p+2}+w_{n, m-1, p+2}}{k^{2}}\right) \\
\left(1+2 r^{2}\right) u_{n, m, p+2}-r^{2}\left(u_{n, m+1, p+2}+u_{n, m-1, p+2}\right)=-z^{2} \delta^{2} \sin \left(u_{n, m, p}-w_{n, m, p}\right) \\
-\left(1+2 r^{2}\right) u_{n, m, p}+r^{2}\left(u_{n+1, m, p}+u_{n-1, m, p}\right)+2 u_{n, m, p+1} \\
\left(1+2 c^{2} r^{2}\right) w_{n, m, p+2}-c^{2} r^{2}\left(w_{n, m+1, p+2}+w_{n, m-1, p+2}\right)
\end{array}\right) z^{2} \sin \left(u_{n, m, p}-w_{n, m, p}\right) \\
\ldots(17) \quad-\left(1+2 c^{2} r^{2}\right) w_{n, m, p}+c^{2} r^{2}\left(w_{n+1, m, p}+w_{n-1, m, p}\right)+2 w_{n, m, p+1}
\end{array}
\end{gathered}
$$

المعادلات (16) و (17) تمثل صيغة أل (ADI) لنظام Sine-Gordon ،أما بالنسبة للنقاط في المستوي 2 (2)

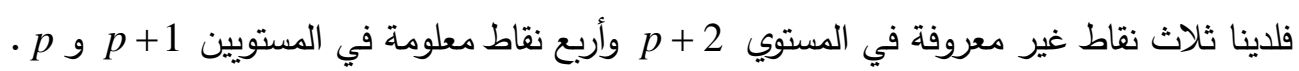
المعادلات (16) و(17) تكوّن كذلك نظام جبري ثلاثي الأقطار

$\mathrm{AX}=\mathrm{B}$

$\mathrm{VX}=\mathrm{C}$

الشروط الحدودية تستخدم في المعادلة الأولى والأخيرة فحسب وكما يأتي:-

$$
\begin{array}{ll}
u_{n, 1, p+2}=0 & g \quad u_{n, M, p+2}=0 \\
w_{n, 1, p+2}=0 & g w_{n, M, p+2}=0
\end{array}
$$

\section{Fourier (Von-Neumann)}

\section{5. تحليل الاستقرارية لطريقة الـ (ADE) لنظام Sine-Gordon باستخدام طريقة}

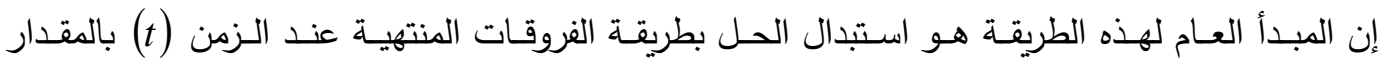

$$
i=\sqrt{-1} \quad \text { حيث } \gamma^{p} e^{i \beta n h} e^{i \alpha m k} \quad, \quad \zeta^{p} e^{i \beta n h} e^{i \alpha m k}
$$

$x=n h, y=m k, t=p z, h=\Delta x, k=\Delta y, z=\Delta t, \alpha>0, \beta>0, \sigma>0$

$u_{n, m, p}=e^{\sigma p z} e^{i \beta n h} e^{i \alpha m k}=\left(e^{\sigma z}\right)^{p} e^{i \beta n h} e^{i \alpha m k}=\zeta^{p} e^{i \beta n h} e^{i \alpha m k}$

لتطبيق طريقة Von-Neumann على النظام (1) نقوم بإهمال الحد اللاخطي منه وباستخدام طريقة الـ ADE

$u_{n, m, p+1}=-u_{n, m, p-1}+\left(2-4 r^{2}\right) u_{n, m, p}+r^{2}\left(u_{n+1, m, p}+u_{n-1, m, p}+u_{n, m+1, p}+u_{n, m-1, p}\right)$

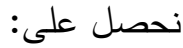

$w_{n, m, p+1}=-w_{n, m, p-1}+\left(2-4 c^{2} r^{2}\right) w_{n, m, p}+c^{2} r^{2}\left(w_{n+1, m, p}+w_{n-1, m, p}+w_{n, m+1, p}+w_{n, m-1, p}\right)$

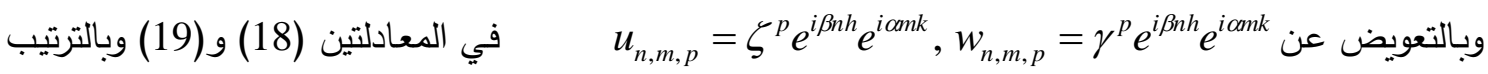




$$
\begin{aligned}
& \zeta^{p+1} e^{i \beta n h} e^{i \alpha m k}=-\zeta^{p-1} e^{i \beta n h} e^{i \alpha m k}+\left(2-4 r^{2}\right) \zeta^{p} e^{i \beta n h} e^{i \alpha m k} \\
& +r^{2}\left(\zeta^{p} e^{i \beta(n+1) h} e^{i \alpha m k}+\zeta^{p} e^{i \beta(n-1) h} e^{i \alpha m k}+\zeta^{p} e^{i \beta n h} e^{i \alpha(m+1) k}+\zeta^{p} e^{i \beta n h} e^{i \alpha(m-1) k}\right) \\
& \gamma^{p+1} e^{i \beta n h} e^{i \alpha m k}=-\gamma^{p-1} e^{i \beta n h} e^{i \alpha m k}+\left(2-4 r^{2} c^{2}\right) \gamma^{p} e^{i \beta n h} e^{i \alpha m k} \\
& +c^{2} r^{2}\left(\gamma^{p} e^{i \beta(n+1) h} e^{i \alpha m k}+\gamma^{p} e^{i \beta(n-1) h} e^{i \alpha m k}+\gamma^{p} e^{i \beta n h} e^{i \alpha(m+1) k}+\gamma^{p} e^{i \beta n h} e^{i \alpha(m-1) k}\right) \\
& \text { وبقسمة المعادلتين أعلاه على } \gamma^{p} e^{i \beta n h} e^{i \alpha m k} \zeta^{p} e^{i \beta n h} e^{i \alpha m k} \\
& \zeta=-\zeta^{-1}+\left(2-4 r^{2}\right)+r^{2}\left(e^{i \beta h}+e^{-i \beta h}+e^{i \alpha k}+e^{-i \alpha k}\right) \\
& \gamma=-\gamma^{-1}+\left(2-4 c^{2} r^{2}\right)+c^{2} r^{2}\left(e^{i \beta h}+e^{-i \beta h}+e^{i \alpha k}+e^{-i \alpha k}\right) \\
& \Rightarrow \frac{\zeta^{2}+1}{\zeta}=\left(2-4 r^{2}\right)+r^{2}(2 \cos (\beta h)+2 \cos (\alpha k)) \\
& \Rightarrow \frac{\gamma^{2}+1}{\gamma}=\left(2-4 c^{2} r^{2}\right)+c^{2} r^{2}(2 \cos (\beta h)+2 \cos (\alpha k)) \\
& \Rightarrow \frac{\zeta^{2}+1}{\zeta}=2-4 r^{2}+2 r^{2}\left[1-2 \sin ^{2}\left(\frac{\beta h}{2}\right)+1-2 \sin ^{2}\left(\frac{\alpha k}{2}\right)\right] \\
& \Rightarrow \frac{\gamma^{2}+1}{\gamma}=2-4 c^{2} r^{2}+2 c^{2} r^{2}\left[1-2 \sin ^{2}\left(\frac{\beta h}{2}\right)+1-2 \sin ^{2}\left(\frac{\alpha k}{2}\right)\right] \\
& \Rightarrow \frac{\zeta^{2}+1}{\zeta}=2\left[1-2 r^{2} \sin ^{2}\left(\frac{\beta h}{2}\right)-2 r^{2} \sin ^{2}\left(\frac{\alpha k}{2}\right)\right] \\
& \Rightarrow \frac{\gamma^{2}+1}{\gamma}=2\left[1-2 c^{2} r^{2} \sin ^{2}\left(\frac{\beta h}{2}\right)-2 c^{2} r^{2} \sin ^{2}\left(\frac{\alpha k}{2}\right)\right] \\
& \Rightarrow \frac{\zeta^{2}+1}{\zeta}=2 A \\
& \Rightarrow \frac{\gamma^{2}+1}{\gamma}=2 B
\end{aligned}
$$$$
A=\left[1-2 r^{2} \sin ^{2}\left(\frac{\beta h}{2}\right)-2 r^{2} \sin ^{2}\left(\frac{\alpha k}{2}\right)\right]
$$$$
\mathbf{B}=\left[1-2 c^{2} r^{2} \sin ^{2}\left(\frac{\beta h}{2}\right)-2 c^{2} r^{2} \sin ^{2}\left(\frac{\alpha k}{2}\right)\right]
$$$$
\Rightarrow \zeta^{2}-2 \zeta A+1=0
$$$$
\Rightarrow \gamma^{2}-2 B \gamma+1=0
$$$$
\Rightarrow \zeta=A \mp \sqrt{A-1}
$$$$
\Rightarrow \gamma=B \mp \sqrt{B-1}
$$

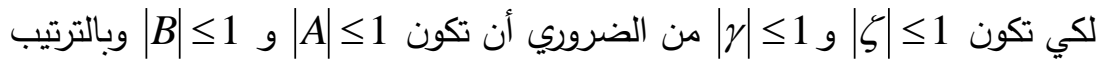

$\Rightarrow\left|1-2 r^{2} \sin ^{2}\left(\frac{\beta h}{2}\right)-2 r^{2} \sin ^{2}\left(\frac{\alpha k}{2}\right)\right| \leq 1$ 


$$
\begin{aligned}
& \Rightarrow\left|1-2 c^{2} r^{2} \sin ^{2}\left(\frac{\beta h}{2}\right)-2 c^{2} r^{2} \sin ^{2}\left(\frac{\alpha k}{2}\right)\right| \leq 1 \\
& -1 \leq 1-2 r^{2} \sin ^{2}\left(\frac{\beta h}{2}\right)-2 r^{2} \sin ^{2}\left(\frac{\alpha k}{2}\right) \leq 1 \\
& 1-2 r^{2} \sin ^{2}\left(\frac{\beta h}{2}\right)-2 r^{2} \sin ^{2}\left(\frac{\alpha k}{2}\right) \leq 1 \\
& -2 r^{2}\left(\sin ^{2}\left(\frac{\beta h}{2}\right)+\sin ^{2}\left(\frac{\alpha k}{2}\right)\right) \leq 0 \\
& r^{2}\left(\sin ^{2}\left(\frac{\beta h}{2}\right)+\sin ^{2}\left(\frac{\alpha k}{2}\right)\right) \geq 0
\end{aligned}
$$

وبأخذ المتباينة (20)

$$
\sin ^{2}\left(\frac{\alpha k}{2}\right)=1 \text { لبعض قيم } \sin ^{2}\left(\frac{\beta h}{2}\right)=1 \text { و يكون } 1 \text { يكو }
$$

$r^{2} \geq 0$

وهذا صحيح دائما. وبأخذ الطرف الأيسر للمتباينة نحصل على :-

$-1 \leq 1-2 r^{2} \sin ^{2}\left(\frac{\beta h}{2}\right)-2 r^{2} \sin ^{2}\left(\frac{\alpha k}{2}\right)$

$-2 \leq-2 r^{2}\left(\sin ^{2}\left(\frac{\beta h}{2}\right)+\sin ^{2}\left(\frac{\alpha k}{2}\right)\right)$

$r^{2}\left(\sin ^{2}\left(\frac{\beta h}{2}\right)+\sin ^{2}\left(\frac{\alpha k}{2}\right)\right) \leq 1$

$r^{2} \leq \frac{1}{\sin ^{2}\left(\frac{\beta h}{2}\right)+\sin ^{2}\left(\frac{\alpha k}{2}\right)}$

$\frac{z^{2}}{h^{2}} \leq \frac{1}{\sin ^{2}\left(\frac{\beta h}{2}\right)+\sin ^{2}\left(\frac{\alpha k}{2}\right)}$

$z^{2} \leq \frac{h^{2}}{\sin ^{2}\left(\frac{\beta h}{2}\right)+\sin ^{2}\left(\frac{\alpha k}{2}\right)}$

$z^{2} \leq \frac{h^{2}}{2}$

وعليه فإنّ الطريقة تكون مستقرة تحت الثرط

وبنفس الأسلوب يجرى على المتباينة (21) فنحصل على

$c^{2} r^{2} \geq 0$ 
وعليه فإنّ الطريقة تكون مستقرة تحت الشرط $z^{2} \leq \frac{h^{2}}{2 c^{2}}$

6. تحليل الاستقرارية لطريقة الـ (ADI) لنظام Sine-Gordon باستخدام طريقة

\section{:Fourier(Von-Neumann)}

سـنقوم أولا بدراسـة استقرارية الخطوة الأولـى لطريقـة الـ ADI، أي الحركـة مـن المستوى 1- 1 إلـى المستوى 1 p+1] [6]، [11]) .وباستخدام طريقة الـ للنظام (1) بعد إهمال الحد اللاخطي منه نحصل على: $\left(1+2 r^{2}\right) u_{n, m, p+1}-r^{2}\left(u_{n+1, m, p+1}+u_{n-1, m, p+1}\right)=$

$\left(1+2 c^{2} r^{2}\right) w_{n, m, p+1}-c^{2} r^{2}\left(w_{n+1, m, p+1}+w_{n-1, m, p+1}\right)=$ $\ldots(22)-\left(1+2 r^{2}\right) u_{n, m, p-1}+r^{2}\left(u_{n, m+1, p-1}+u_{n, m-1, p-1}\right)+2 u_{n, m, p}$ $\ldots(23)-\left(1+2 c^{2} r^{2}\right) w_{n, m, p-1}+c^{2} r^{2}\left(w_{n, m+1, p-1}+w_{n, m-1, p-1}\right)+2 w_{n, m, p}$

وبالتعويض عن $\quad u_{n, m, p}=\zeta^{p} e^{i \beta n h} e^{i \alpha m k}, w_{n, m, p}=\gamma^{p} e^{i \beta n h} e^{i \alpha m k}$ في المعادلتين (22) و(23) وبالترتيـب $\left(1+2 r^{2}\right) \zeta^{p+1} e^{i \beta n h} e^{i \alpha m k}-r^{2}\left(\zeta^{p+1} e^{i \beta(n+1) h} e^{i \alpha m k}+\zeta^{p+1} e^{i \beta(n-1) h} e^{i \alpha m k}\right)$

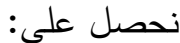

$$
\begin{aligned}
& =-\left(1+2 r^{2}\right) \zeta^{p-1} e^{i \beta n h} e^{i \alpha m k}+r^{2}\left(\zeta^{p-1} e^{i \beta n h} e^{i \alpha(m+1) k}+\zeta^{p-1} e^{i \beta n h} e^{i \alpha(m-1) k}\right)+2 \zeta^{p} e^{i \beta n h} e^{i \alpha m k} \\
& \left(1+2 c^{2} r^{2}\right) \gamma^{p+1} e^{i \beta n h} e^{i \alpha m k}-c^{2} r^{2}\left(\gamma^{p+1} e^{i \beta(n+1) h} e^{i \alpha m k}+\gamma^{p+1} e^{i \beta(n-1) h} e^{i \alpha m k}\right) \\
& =-\left(1+2 c^{2} r^{2}\right) \gamma^{p-1} e^{i \beta n h} e^{i \alpha m k}+c^{2} r^{2}\left(\gamma^{p-1} e^{i \beta n h} e^{i \alpha(m+1) k}+\gamma^{p-1} e^{i \beta n h} e^{i \alpha(m-1) k}\right)+2 \gamma^{p} e^{i \beta n h} e^{i \alpha m k} \\
& \text { وبقسمة المعادلتين أعلاه على } \gamma^{p} e^{i \beta n h} e^{i \alpha m k} \zeta^{p} e^{i \beta n h} e^{i \alpha m k} \\
& \left(1+2 r^{2}\right) \zeta-r^{2}\left(\zeta e^{i \beta h}+\zeta e^{-i \beta h}\right)=-\left(1+2 r^{2}\right) \zeta^{-1}+r^{2}\left(\zeta^{-1} e^{i \alpha k}+\zeta^{-1} e^{-i \alpha k}\right)+2 \\
& \left(1+2 c^{2} r^{2}\right) \gamma-c^{2} r^{2}\left(\gamma e^{i \beta h}+\gamma e^{-i \beta h}\right)=-\left(1+2 c^{2} r^{2}\right) \gamma^{-1}+c^{2} r^{2}\left(\gamma^{-1} e^{i \alpha k}+\gamma^{-1} e^{-i \alpha k}\right)+2 \\
& \left(1+2 r^{2}\right)\left(\zeta+\zeta^{-1}\right)-2=r^{2}\left(\zeta\left(e^{i \beta h}+e^{-i \beta h}\right)\right)+r^{2}\left(\zeta^{-1}\left(e^{i \alpha k}+e^{-i \alpha k}\right)\right) \\
& \left(1+2 c^{2} r^{2}\right)\left(\gamma+\gamma^{-1}\right)-2=c^{2} r^{2}\left(\gamma\left(e^{i \beta h}+e^{-i \beta h}\right)\right)+c^{2} r^{2}\left(\gamma^{-1}\left(e^{i \alpha k}+e^{-i \alpha k}\right)\right) \\
& \left(1+2 r^{2}\right)\left(\zeta+\zeta^{-1}\right)-2=2 r^{2}\left(\zeta+\zeta^{-1}\right)-4 r^{2} \zeta \sin ^{2}\left(\frac{\beta h}{2}\right)-4 r^{2} \zeta^{-1} \sin ^{2}\left(\frac{\alpha k}{2}\right) \\
& \left(1+2 c^{2} r^{2}\right)\left(\gamma+\gamma^{-1}\right)-2=2 c^{2} r^{2}\left(\gamma+\gamma^{-1}\right)-4 c^{2} r^{2} \gamma \sin ^{2}\left(\frac{\beta h}{2}\right)-4 c^{2} r^{2} \gamma^{-1} \sin ^{2}\left(\frac{\alpha k}{2}\right) \\
& \left(\zeta+\zeta^{-1}\right)\left(1+2 r^{2}-2 r^{2}\right)+4 r^{2} \zeta \sin ^{2}\left(\frac{\beta h}{2}\right)+4 r^{2} \zeta^{-1} \sin ^{2}\left(\frac{\alpha k}{2}\right)-2=0 \\
& \left(\gamma+\gamma^{-1}\right)\left(1+2 c^{2} r^{2}-2 c^{2} r^{2}\right)+4 c^{2} r^{2} \gamma \sin ^{2}\left(\frac{\beta h}{2}\right)+4 c^{2} r^{2} \gamma^{-1} \sin ^{2}\left(\frac{\alpha k}{2}\right)-2=0 \\
& \zeta^{2}+1+4 r^{2} \zeta^{2} \sin ^{2}\left(\frac{\beta h}{2}\right)+4 r^{2} \sin ^{2}\left(\frac{\alpha k}{2}\right)-2 \zeta=0 \\
& \gamma^{2}+1+4 c^{2} r^{2} \gamma^{2} \sin ^{2}\left(\frac{\beta h}{2}\right)+4 c^{2} r^{2} \sin ^{2}\left(\frac{\alpha k}{2}\right)-2 \gamma=0
\end{aligned}
$$




$$
\begin{aligned}
& \zeta^{2}\left(1+4 r^{2} \sin ^{2}\left(\frac{\beta h}{2}\right)\right)-2 \zeta+\left(1+4 r^{2} \sin ^{2}\left(\frac{\alpha k}{2}\right)\right)=0 \\
& \gamma^{2}\left(1+4 c^{2} r^{2} \sin ^{2}\left(\frac{\beta h}{2}\right)\right)-2 \gamma+\left(1+4 c^{2} r^{2} \sin ^{2}\left(\frac{\alpha k}{2}\right)\right)=0 \\
& \zeta^{2}-\left(\frac{2}{1+4 r^{2} \sin ^{2}\left(\frac{\beta h}{2}\right)}\right) \zeta+\left(\frac{1+4 r^{2} \sin ^{2}\left(\frac{\alpha k}{2}\right)}{1+4 r^{2} \sin ^{2}\left(\frac{\beta h}{2}\right)}\right)=0 \\
& \gamma^{2}-\left(\frac{2}{1+4 c^{2} r^{2} \sin ^{2}\left(\frac{\beta h}{2}\right)}\right) \gamma+\left(\frac{1+4 c^{2} r^{2} \sin ^{2}\left(\frac{\alpha k}{2}\right)}{1+4 c^{2} r^{2} \sin ^{2}\left(\frac{\beta h}{2}\right)}\right)=0
\end{aligned}
$$

$$
\begin{gathered}
H=\frac{1}{1+4 r^{2} \sin ^{2}\left(\frac{\beta h}{2}\right)} \quad, \quad G=\frac{1+4 r^{2} \sin ^{2}\left(\frac{\alpha k}{2}\right)}{1+4 r^{2} \sin ^{2}\left(\frac{\beta h}{2}\right)} \\
I=\frac{1}{1+4 c^{2} r^{2} \sin ^{2}\left(\frac{\beta h}{2}\right)} \quad, \quad J=\frac{1+4 c^{2} r^{2} \sin ^{2}\left(\frac{\alpha k}{2}\right)}{1+4 c^{2} r^{2} \sin ^{2}\left(\frac{\beta h}{2}\right)}
\end{gathered}
$$

$$
\zeta^{2}-2 H \zeta+G=0
$$$$
\gamma^{2}-2 I \gamma+J=0
$$$$
\zeta=H \mp \sqrt{H^{2}-G}
$$$$
\gamma=I \mp \sqrt{I^{2}-J}
$$

$$
\text { الآن إذا كان }
$$

$$
\zeta=H \mp i \sqrt{G-H^{2}}
$$$$
\gamma=I \mp i \sqrt{J-I^{2}}
$$$$
|\zeta|=(G)^{\frac{1}{2}}
$$$$
|\gamma|=(J)^{\frac{1}{2}}
$$

$$
\text { ولبعض القيم لـ } \beta \text { ر تكون كل من =1, =1, }
$$

$|\zeta|=1$

$|\gamma|=1$

وعليه فإنّ الخطوة الأولى تكون مستقرة بدون شروط.

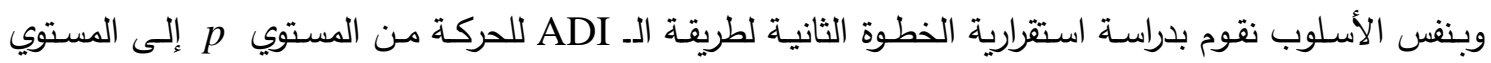
p+2 ونستتج أن الخطوة الثانية تكون مستقرة أيضاً بدون شرط. 
لغرض الحل العددي نأخذ نظام Sine-Gordon في بعدين والمتمثل بالنظام (1)

$$
\begin{aligned}
& \frac{\partial^{2} u}{\partial t^{2}}-\frac{\partial^{2} u}{\partial x^{2}}-\frac{\partial^{2} u}{\partial y^{2}}=-\delta^{2} \sin (u-w) \\
& \frac{\partial^{2} w}{\partial t^{2}}-c^{2} \frac{\partial^{2} w}{\partial x^{2}}-c^{2} \frac{\partial^{2} w}{\partial y^{2}}=\sin (u-w)
\end{aligned}
$$

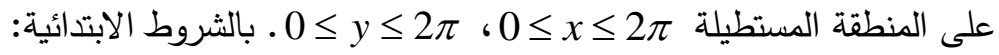

$$
\begin{array}{lll}
u(x, y, 0)=f(x, y), & \frac{\partial u(x, y, 0)}{\partial t}=0, & 0 \leq x, y \leq 2 \pi \\
w(x, y, 0)=g(x, y) \quad, \quad \frac{\partial w(x, y, o)}{\partial t}=0 & , \quad 0 \leq x, y \leq 2 \pi
\end{array}
$$

والشرط الحدودي:

\begin{tabular}{|c|c|c|c|}
\hline \multicolumn{2}{|c|}{$\begin{array}{c}\text { ADE Method (fourth level) } \\
\mathrm{x}=1.88495559 \\
\mathrm{~h}=0.31415927 \\
\mathrm{k}=0.31415927 \\
\mathrm{z}=0.22214415 \\
\mathrm{c}=0.5 \\
\delta=1\end{array}$} & \multicolumn{2}{|c|}{$\begin{array}{c}\text { ADI Method (fourth level) } \\
\begin{array}{c}\mathrm{x}=1.88495559 \\
\mathrm{~h}=0.31415927 \\
\mathrm{k}=0.31415927 \\
\mathrm{z}=0.22214415 \\
\mathrm{c}=0.5 \\
\delta=1\end{array}\end{array}$} \\
\hline$u(x, y, t)$ & $w(x, y, t)$ & $u(x, y, t)$ & $w(x, y, t)$ \\
\hline 0 & 0 & 0 & 0 \\
\hline 0.2838 & 0.2911 & 0.2871 & 0.2921 \\
\hline 0.5399 & 0.5536 & 0.5461 & 0.5556 \\
\hline 0.7431 & 0.7620 & 0.7517 & 0.7647 \\
\hline 0.8736 & 0.8958 & 0.8837 & 0.8989 \\
\hline 0.9185 & 0.9419 & 0.9291 & 0.9452 \\
\hline 0.8736 & 0.8958 & 0.8837 & 0.8989 \\
\hline 0.7431 & 0.7620 & 0.7517 & 0.7647 \\
\hline 0.5399 & 0.5536 & 0.5461 & 0.5556 \\
\hline 0.2838 & 0.2911 & 0.2871 & 0.2921 \\
\hline 0 & 0 & 0 & 0 \\
\hline-0.2838 & -0.2911 & -0.2871 & -0.2921 \\
\hline-0.5399 & -0.5536 & -0.4561 & -0.5556 \\
\hline-0.7431 & -0.7620 & -0.7517 & -0.7647 \\
\hline
\end{tabular}

$u(x, y, 0)=\sin (x) * \sin (y)$

$w(x, y, 0)=\sin (x) * \sin (y)$

جدول (1). مقارنة بين طريقتي الـ ADE و الـ ADI 


\begin{tabular}{|c|c|c|c|}
\hline-0.8736 & -0.8958 & -0.8837 & -0.8989 \\
\hline-0.9185 & -0.9419 & -0.9291 & -0.9452 \\
\hline-0.8736 & -0.8958 & -0.8837 & -0.8989 \\
\hline-0.7431 & -0.7620 & -0.7517 & -0.7647 \\
\hline-0.5399 & -0.5536 & -0.5461 & -0.5556 \\
\hline-0.2838 & -0.2911 & -0.2871 & -0.2921 \\
\hline 0 & 0 & 0 & 0 \\
\hline
\end{tabular}

من خلال ملاحظة النتائج نستنتج أن طريقة الـ ADI هي أفضل من طريقة الـ ADE في بعدين كما أن

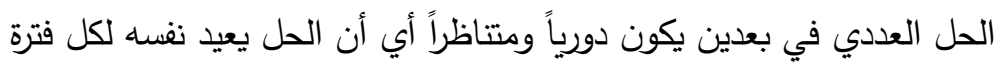
ولذلك فإننا نحتاج إلى حسابات أقل وبالتالي إلى وقدانت أقلى

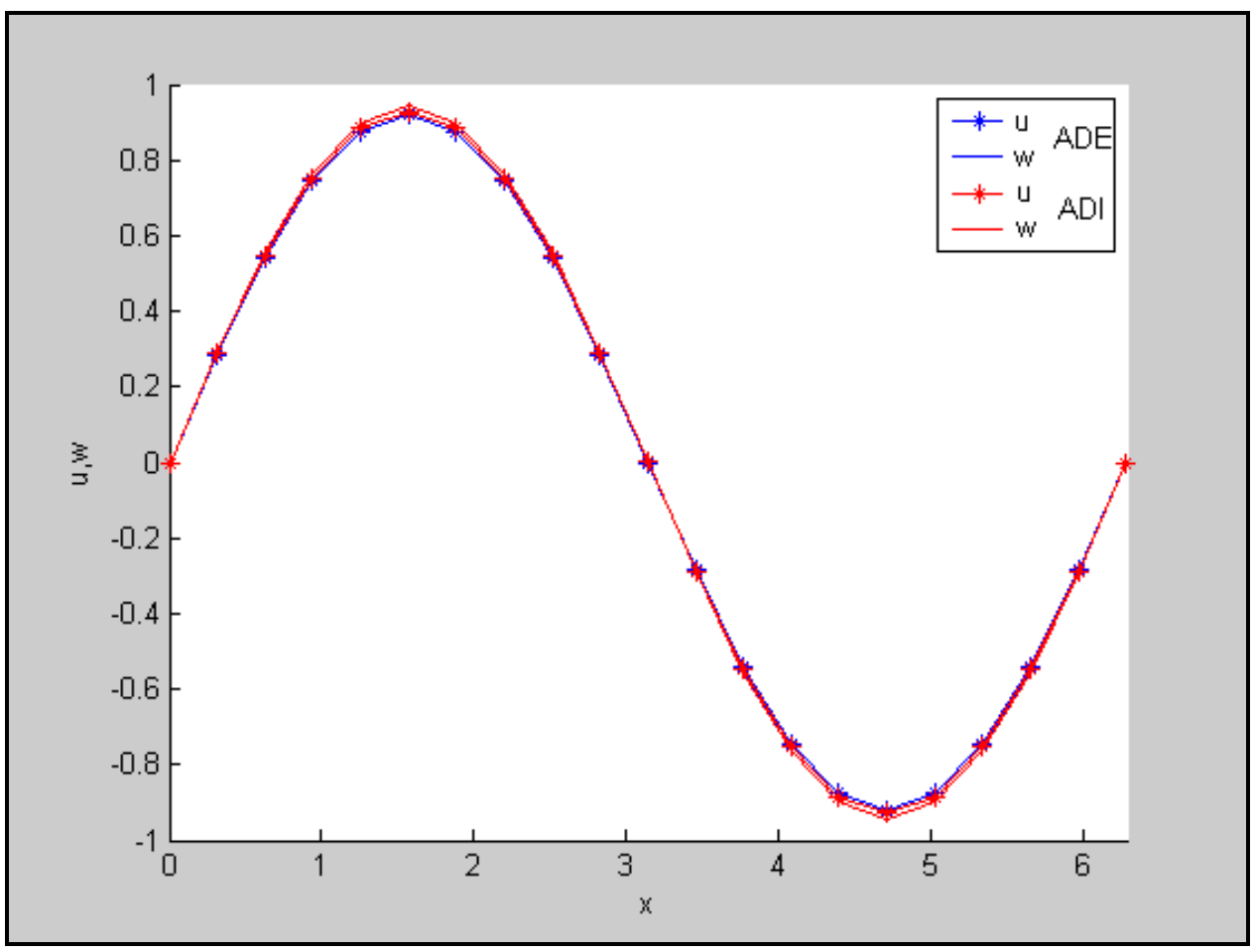

$$
\text { شكل (1). مقارنة بين طريقتي الـ ADE والـ ADI عندما }
$$

8. الاستنتاجات:

من خـلال المقارنـة بين حل نظام Sine-Gordon في بعدين فقد تبين أنّ حل نظـام Sine-Gordon كمام

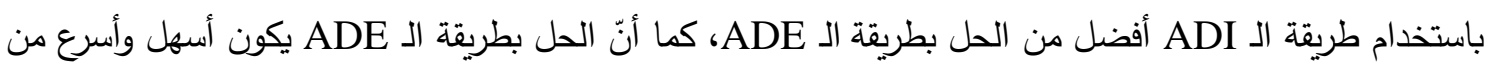
الحل بطريقة الـ ADI، وأنّ الحل بكلتا الطريقتين يكون متتاظرا مما يؤدي الى توفير الوقت والجهد، وتم تحليل

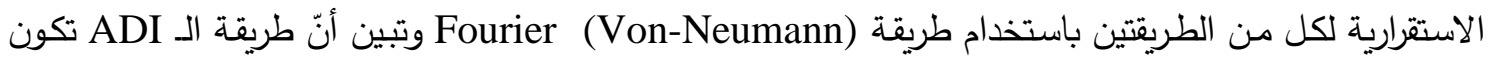
مستقرة لجميع قيم الم أي انها تكون مستقرة على نحو غير مشروط في حين طريقة الـ ADE تكون مستقرة تحت

$$
\text { الشرط }
$$




$$
\begin{aligned}
& \text { المصادر } \\
& \text { الدلفي، حسن مجيد حسون ومشكور، محمود عطا الله مشكور ، (1999)، "التحليل الهندسي والعددي } \\
& \text { التطبيقي"، الجامعة التكنلوجية، بغداد. } \\
& \text { العاني، عطا الله ثامر العاني، (1982)، "المعادلات التفاضلية الجزئية للكليات العلمية_والهندسية"، } \\
& \text { جامعة بغداد. } \\
& \text { شكر ، نزار حمدون، (1989)، "مسائل القيم الحدودية"، جامعة الموصل. }
\end{aligned}
$$

[4] Ablowitz, M.J., Herbst, B.M. and Schober, C., (1996), "On the numerical solution of the Sine-Gordon equation: I, Integrable discretizations and howoclinic manifolds", J. Compnt. Phys. 126, pp.299-314.

[5] Gordon D. Smith (1965); "Numerical Solution of Partial Differential Equations :Finite Difference Methods", second edition, Oxford University press.

[6] Jain, M.K., Iyengar, S. R.K., and Jain, R.K., (1985); "Numerical Methods for Scientific and Engineering Computation", second edition, Wiley Eastern Limited.

[7] Jesus Adrian Espinola Rocha, (2000), "Some exact solutions of a coupled system of Klein-Gordon eqations", The nonlinear journal ,Vol.2, PP.1-13.

[8] Khomeriki, R., and Leon, J., (2005), "Bistability in Sine-Gordon:The ideal switch", Phys. Rev. E71, 056620.

[9] Lu, X., and Schmid, R., (1998), " Symplectic integration of Sine-Gordon type systems", IMACS international conference MODELLING 98, Prague, July 711.

[10] Peaceman, D. W. and Rechford, H.H., (1955), "The numerical solution of Parabolic and Elliptic Differential Equations", SIAM J., 3, P.28

[11] Shanthakumar, M., (1989); "Computer Based Numerical Analysis", Khanna Publishers. 